\title{
Young solar type active stars: the TYC 2627-638-1 system ${ }^{\star, \star \star, \star \star \star}$
}

\author{
K. Oláh ${ }^{1}$, H. Korhonen ${ }^{2}$, K. Vida ${ }^{1}$, I. Ilyin ${ }^{3}$, T. H. Dall ${ }^{2}$, S. P. Järvinen ${ }^{3}$, J. Jurcsik ${ }^{1}$, M. I. Andersen ${ }^{4}$, A. A. Djupvik ${ }^{5}$, \\ T. Pursimo ${ }^{5}$, A. Moór ${ }^{1}$, J. Datson ${ }^{5}$, R. Karjalainen ${ }^{5}$, T. Liimets ${ }^{5,6}$, J. Kubát ${ }^{7}$, and A. Kawka ${ }^{7}$ \\ 1 Konkoly Observatory of the Hungarian Academy of Sciences, 1525 Budapest, Hungary \\ e-mail: olah@konkoly.hu \\ 2 ESO, Karl-Schwarzschild Strasse 2, 85748 Garching bei Munchen, Germany \\ Astrophysical Institute Potsdam, An der Sternwarte 16, 14482 Potsdam, Germany \\ 4 Dark Cosmology Centre, Niels Bohr Institute, University of Copenhagen, Juliane Mariesvej 30, 2100 Kbh Ø, Denmark \\ 5 Nordic Optical Telescope, Apartado 474, 38700 Santa Cruz de La Palma, Santa Cruz de Tenerife, Spain \\ 6 Tartu Observatoorium, Tõravere 61602, Estonia \\ 7 Astronomický ústav, Akademie věd České republiky, 25165 Ondřejov, Czech Republic
}

Received 14 July 2009 / Accepted 6 March 2010

\section{ABSTRACT}

\begin{abstract}
We present $B V(R I)_{\mathrm{C}}$ and $J H K$ s photometry and low- and high-resolution spectroscopy of the 11th mag G-type star TYC 2627-638-1. Our investigation reveals that the target is separated into two young, early-G-type main-sequence (or late pre-main-sequence) stars, which are most probably bound and form a wide binary system. A substellar body orbits the brighter component as implied by radial velocity variations. The brighter component possibly also has a faint, later type stellar companion.

Both components of the wide binary have clear emission cores in the $\mathrm{Ca}$ II $\mathrm{H} \& \mathrm{~K}$ lines and filled-in $\mathrm{H} \alpha$ absorption, indicating that both stars are chromospherically active. Our photometric time series reveals clear but only a few hundredths of a magnitude amplitude rotational modulation, which is most likely due to cool starspots. Two distinct periods, near 3.5 and 3.7 days, are found in the brightness variations. Photometry obtained separately of the two components of the wide binary show that these periodicities belong to the brighter star. The fainter component shows a much slower light variation of about 0.3 mag. amplitude. In addition, long-term changes in the brightness of both stars are seen. The spectral energy distribution shows a strong near-infrared excess in the fainter component of the wide binary.
\end{abstract}

Key words. stars: activity - stars: atmospheres - stars: late-type - starspots

\section{Introduction}

With the increasing accuracy of photometric measurements and due to several survey programmes on and beyond Earth, the number of the newly discovered variable stars is increasing enormously every day. Indeed, new variables cannot be properly catalogued in a universal system. Therefore the "discovery" of a new variable star in the field of a well-observed object means usually just a warning: the star cannot be used as a comparison. However, from time to time interesting new variables show up in some well-observed fields. The real advantage of these kinds of serendipitously uncovered variables is that usually a great deal of data have been taken, with good standard and comparison stars for the sake of the main project.

Studying variability of solar type stars of different ages is especially important, and even more interesting are the cases when two such stars are bound together. The object studied in this paper was found to be variable in the course of monitoring the Blazhko-RR Lyr star MW Lyr, see Jurcsik et al. (2008) for

* Based on the observations obtained at the Nordic Optical Telescope, Observatorio Roque de los Muchachos, La Palma, Canary Islands, Spain.

$\star \star$ Based on observations collected with the automatic $60-\mathrm{cm}$ and the 1-m telescopes of Konkoly Observatory, Hungary.

$\star \star \star$ The photometric data are available in electronic form at the CDS via anonymous ftp to cdsarc.u-strasbg. fr $(130.79 .128 .5)$ or via http://cdsweb.u-strasbg.fr/cgi-bin/qcat?J/A+A/515/A81 more details. It consists of two G-dwarf components very close to each other on the sky. We report light variations of these stars on long timescale as well as rotational modulation of variable amplitudes and $\mathrm{Ca}$ II H\&K emission of both components. Below we give a detailed analysis of our photometric and spectroscopic observations of this interesting and probably binary system.

\section{Observations}

\subsection{Photometric data}

We present $7100 \mathrm{~B}, V, I_{\mathrm{C}}$ and $2391 R_{\mathrm{C}}$ observations, all interpolated to the time of the $V$-magnitude, obtained in 2006-2009 with the automated $60-\mathrm{cm}$ telescope in Budapest and with the 1-m RCC telescope in Piszkéstetô mountain station of Konkoly Observatory, with CCD cameras and $B V(R I)_{\mathrm{C}}$ filters. The equipment and data handling are described in detail in Jurcsik et al. (2008). Data reduction via aperture and PSF photometry was carried out with standard IRAF ${ }^{1}$ packages. Figure 1 shows the finding chart of the brighter companion and the comparison stars as given in Jurcsik et al. (2008).

In Table 1 we give the average magnitudes obtained with the $\mathrm{RCC}$ telescope of the variable and the comparison stars $\mathrm{C} 1, \mathrm{C} 2$,

\footnotetext{
${ }^{1}$ IRAF is distributed by the National Optical Astronomy Observatory, which is operated by the Association of Universities for Research in Astronomy, Inc., under cooperative agreement with the National Science Foundation.
} 

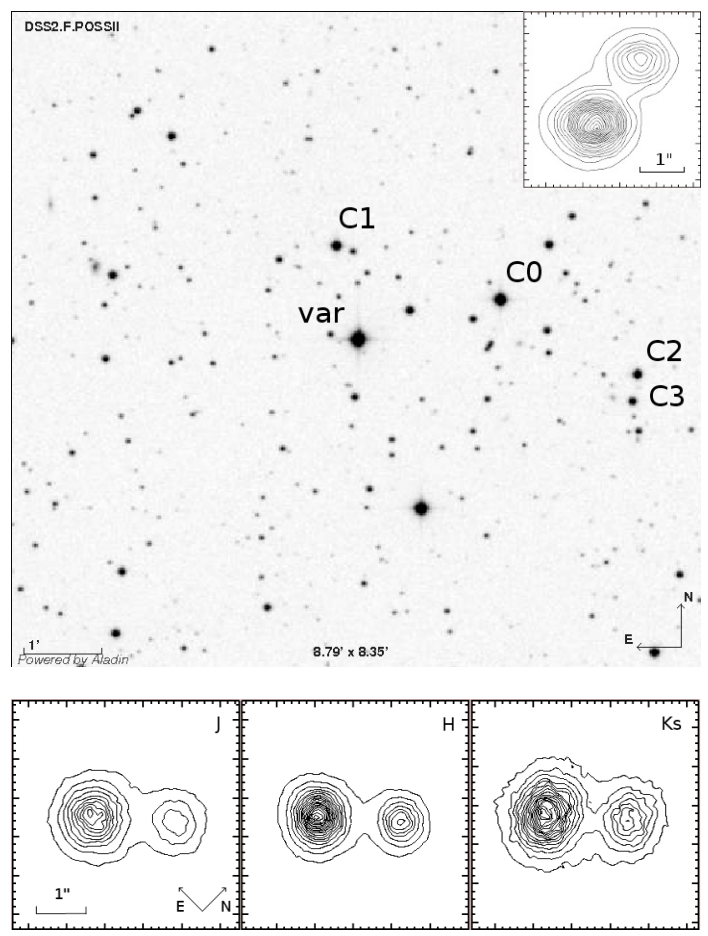

Fig. 1. Finding chart of var = TYC 2627-638-1 and the comparison stars in the field. The top right corner shows that in the position of the variable there are two stars with 1.84 arcsec separation. The contour plot is made from an image made with the $1-\mathrm{m}$ telescope through $I_{\mathrm{C}}$ filter in excellent seeing conditions. Below: NOTCam JHKs images of the two components. Note that the fainter companion star is redder than the variable.

Table 1. Average magnitudes and colour indices of the comparison stars and var = TYC 2627-638-1A+TYC 2627-638-1B (measured together) from the RCC telescope.

\begin{tabular}{lllll}
\hline \hline Star & $V$ & $B-V$ & $V-R_{\mathrm{C}}$ & $V-I_{\mathrm{C}}$ \\
\hline $\mathrm{C} 0$ & 11.841 & 0.582 & 0.315 & 0.666 \\
\hline $\mathrm{C} 1$ & $12.984 \pm .007$ & $0.677 \pm .011$ & $0.374 \pm .010$ & $0.790 \pm .012$ \\
& 12.986 & 0.682 & 0.383 & 0.788 \\
$\mathrm{C} 2$ & $13.908 \pm .011$ & $0.849 \pm .033$ & $0.466 \pm .016$ & $0.959 \pm .024$ \\
& 13.921 & 0.858 & 0.480 & 0.950 \\
PSF $^{*}$ & $13.998 \pm .022$ & $0.891 \pm .030$ & $0.481 \pm .028$ & $0.976 \pm .028$ \\
$\mathrm{C}^{2}$ & $14.364 \pm .017$ & $0.597 \pm .047$ & $0.322 \pm .025$ & $0.681 \pm .030$ \\
& 14.363 & 0.603 & 0.338 & 0.671 \\
\hline var & $10.799 \pm .010$ & $0.639 \pm .016$ & $0.347 \pm .015$ & $0.715 \pm .013$ \\
\hline
\end{tabular}

Notes. Standard values measured by A. Henden from Jurcsik et al. (2008), Table 1, are given in italics. C0 is used as main comparison star. (*) Measured separately from its companion, see text for more details.

and $\mathrm{C} 3$, while we use $\mathrm{C} 0$ as the main comparison. We also give the standard magnitudes of these stars obtained by A. Henden (Jurcsik et al. 2008). It is clear from the results that our photometric system well matches the standard system and that the comparison stars are constant (at least at the level of the accuracy of the measurements), as is well demonstrated in Fig. 7 of Jurcsik et al. (2008).

The top right corner of Fig. 1 shows that close to TYC 2627638-1 another star is found in the north-west direction; the separation between the two stars is 1.84 arcsec. The GSC2.3.2 catalogue (2006) includes both TYC 2627-638-1 as N25R000667, and its companion as N25R029219, the latter with $B_{J}=$ 12.42 mag. derived from POSS-II. No further information on the companion star is found in the literature.

This companion is fairly close to the limit of the Tycho- 2 catalogue (Høg et al. 2000). The source density in the Tycho- 2 catalogue in this region of the sky can therefore be used to calculate the probability that the companion is a random star which is not gravitationally bound to TYC 2627-638-1. A Tycho-2 sourcedensity of 92.75 objects per square degree is found, from which we calculate the probability of the pair being a random alignment to less than $0.01 \%$. It is therefore clear beyond any reasonable doubt that these two stars are physically associated. This conclusion is substantially supported by the spectroscopic observations presented later in Sect. 3.3. For the remainder of the paper TYC 2627-638-1 is therefore referred to as TYC 2627-638-1A, while the fainter companion is referred to as TYC 2627-638-1B.

The 2MASS observation (Skrutskie et al. 2006) JHKs magnitudes $(9.678,9.442,9.322$, respectively) contains the light from both components, because according to the catalogue the nearest object is 6.7 arcsec away, whereas the two stars are only 1.84 arcsec from each other. Since the north-west companion TYC 2627-638-1B is redder than the brighter TYC 2627-6381 A (see Fig. 6), the 2MASS JHKs magnitudes contain more and more light from the companion.

On 11 nights (three in 2007, one in 2008 and seven in 2009) we had excellent seeing conditions, and from those observations made with the 1-m telescope the brightness of TYC 2627-638$1 \mathrm{~A}$ and TYC 2627-638-1B was derived in four colours separately with PSF photometry. Average magnitudes, standard deviations and fluxes for the two stars are given in Table 2. Both stars are unresolved in the CCD frames in most nights with the $1-\mathrm{m}$ telescope and throughout all observations with the $60-\mathrm{cm}$ telescope.

Note that the comparison star marked with C2 (Fig. 1) was found to have a close companion as well about 2.8 arcsec to the north and fainter by about $2.5 \mathrm{mag}$, which was measured together with $\mathrm{C} 2$ in most cases with the aperture photometry. From the PSF photometry (i.e., without its companion) C2 is found to be slightly redder (see Table 1), its approximate spectral type is early $\mathrm{K}$ and that of the companion is of mid-F, provided they are Main Sequence stars.

On 2009 November 26 imaging was done with NOTCam ${ }^{2}$ at the Nordic Optical Telescope (NOT) in the $J(1.247 \mu \mathrm{m}), H$ $(1.632 \mu \mathrm{m})$, and $K s(2.140 \mu \mathrm{m})$ bands, using the HR-camera $\left(0.078^{\prime \prime} / \mathrm{pix}\right)$, which yields a field of $80^{\prime \prime}$. In each filter a 9-point dither pattern was made with individual exposure times of 10 and $15 \mathrm{~s}$, giving a total on-source integration time of $135 \mathrm{~s}, 135 \mathrm{~s}$, and $90 \mathrm{~s}$, for $J, H$, and $K \mathrm{~s}$, respectively. The observations were made at an airmass around 2, but the seeing was good enough to resolve the two components in all images ( $F W H M$ varied from $0.6^{\prime \prime}$ to $\left.0.9^{\prime \prime}\right)$. An IRAF script especially written for NOTCam data was used to: 1) flat field correct all images with differential twilight flats from the same night; 2) subtract the sky level with the dithered images to compose sky frames; and 3) shift and add the images to median combine the 9 images per filter. Clouds inhibited an accurate flux calibration, but through the 2MASS magnitudes of the comparison star $\mathrm{C} 1$ (cf. Table 1 and Fig. 1) we arrived at the JHKs magnitudes and fluxes of the two stars given in Table 2.

2 The Nordic Optical Telescope's near-IR camera/spectrograph, see http://www. not.iac.es/instruments/notcam/ for details on the instrument. 
Table 2. Average magnitudes of the two visible components of TYC 2627-638-1 from the RCC telescope and from the NOTCam.

\begin{tabular}{lcccc}
\hline \hline Colour & \multicolumn{2}{c}{ TYC 2627-638-1A } & \multicolumn{2}{c}{ TYC 2627-638-1B } \\
& mag & flux $^{*}$ & mag & flux $^{*}$ \\
\hline$B$ & $11.707 \pm .018$ & 13.9 & $13.21 \pm .21$ & 3.49 \\
$V$ & $11.098 \pm .020$ & 13.6 & $12.45 \pm .16$ & 3.93 \\
$R_{\mathrm{C}}$ & $10.738 \pm .020$ & 11.4 & $12.03 \pm .14$ & 3.49 \\
$I_{\mathrm{C}}$ & $10.378 \pm .014$ & 8.65 & $11.62 \pm .12$ & 2.76 \\
$J^{\dagger}$ & 9.75 & 3.98 & 10.71 & 1.63 \\
$H^{\dagger}$ & 9.55 & 1.68 & 10.49 & 0.71 \\
$K \mathrm{~s}^{\dagger}$ & 9.45 & 0.71 & 10.39 & 0.30 \\
\hline
\end{tabular}

Notes. ${ }^{(*)}$ In units $10^{-14} \mathrm{erg} \mathrm{s} \mathrm{cm}^{-2} / \AA{ }^{(\dagger)}$ estimated conservative error is about \pm 0.15 .

\subsection{Spectroscopic data}

\subsubsection{Low-resolution spectroscopy}

The low-resolution spectroscopic observations used in this study were obtained at the Nordic Optical Telescope using the Andalucia Faint Object Spectrograph and Camera (ALFOSC), which has an E2V Technologies $2 \mathrm{k}$ back illuminated CCD with $13.5-\mu$ pixels.

Two 60 s-long exposures of TYC 2627-638-1A and a 15 slong exposure of a flux standard, BD $+17^{\circ} 4708$, were obtained in the evening of 2006 October 19 using grism \#4 and 1.0 arcsec slit. An order sorter filter GG375 was used to reduce the second order contribution from the blue part of the spectrum, which without a filter would be noticeable redwards from $5900 \AA$. With this filter the second order starts to overlap with the first order around $7500 \AA$. This setup gave a resolving power $(\lambda / \Delta \lambda)$ of about 350 and a spectral coverage of 3550-9100 A. A fringing pattern in the red part of the spectrum starts around $6700 \AA$ and renders the spectra basically useless after $7000 \AA$.

The observations were reduced with the $4 \mathrm{~A}$ reduction package (Ilyin 2000). The reductions included a standard bias subtraction, flat field correction using a halogen lamp and a wavelength calibration using a neon lamp. Unfortunately, neon does not have lines in the blue part of the spectrum and due to this the wavelength solution starts to get progressively worse when going bluewards of $5800 \AA$, and around the $3900 \AA$ the difference to the expected value is approximately $50 \AA$. Still, this drift in the wavelength scale does not affect the identification of the most important lines for the spectral classification. The flux calibration was done with the accurate flux-distribution measurement of $\mathrm{BD}+17^{\circ} 4708$ by Bohlin \& Gilliland (2004). The fluxcalibrated spectrum of the variable is shown in Fig. 2a.

One spectrum around the $\mathrm{H} \alpha$ line, which is a sum of two subsequent 1-h exposures, was taken on 2007 April 13 with a 2-m telescope of the Ondřejov Observatory using a $700 \mathrm{~mm}$ camera of the coudé spectrograph with CCD SiTe ST-005 800×2000 pix with $15-\mu \mathrm{m}$ pixels. For a description of the coudé spectrograph settings see Šlechta \& Škoda (2002). The resulting spectrum is surely a mixture of the light of both components, the seeing conditions in Ondřejov do not allow to resolve such close stars. The spectrum is shown in Fig. 2 b.

\subsubsection{High-resolution spectroscopy}

High-resolution spectra of TYC 2627-638-1A and TYC 2627638-1B were obtained at the Nordic Optical Telescope with FIES high resolution spectrograph with $S / N \sim 70$ for the A
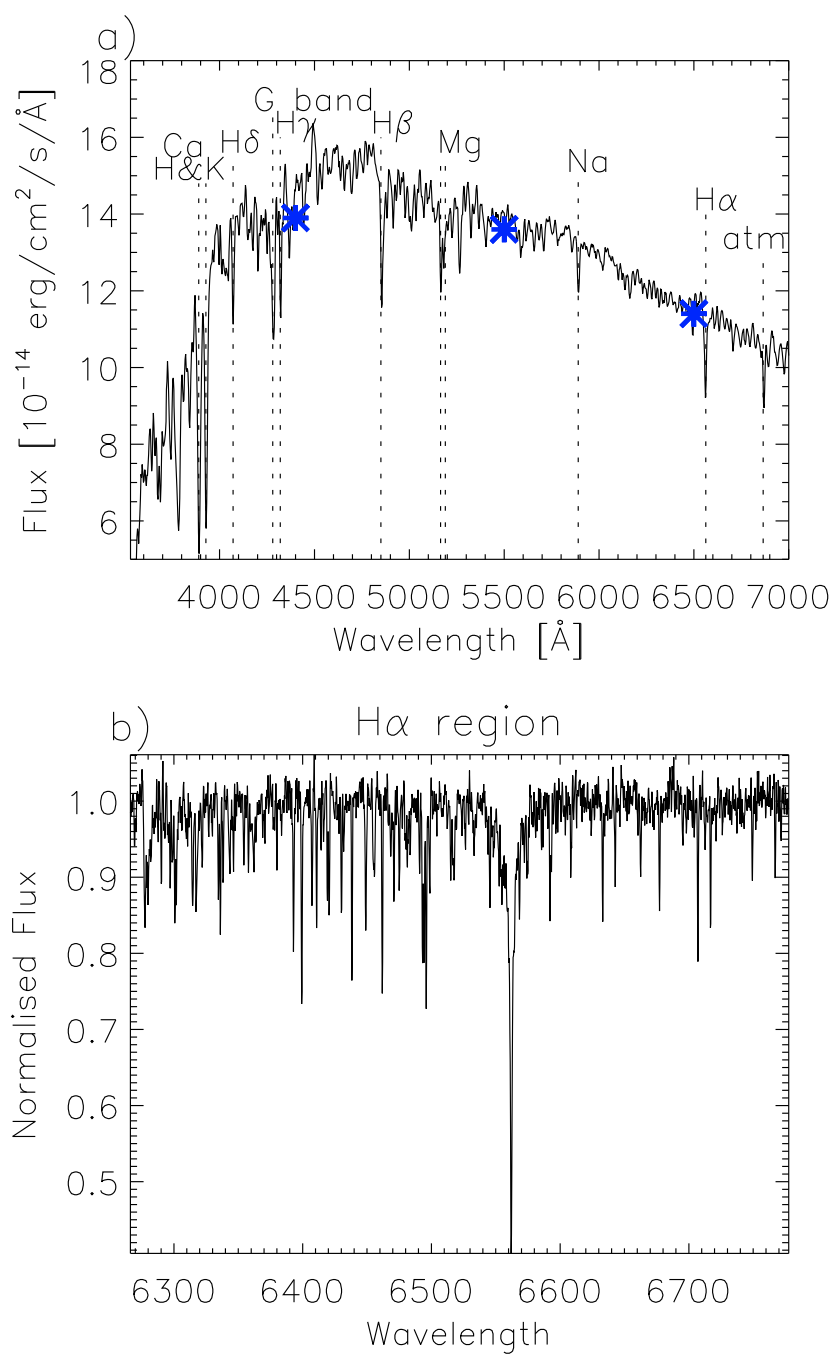

Fig. 2. Spectrum of the new variable. a) The low-resolution ALFOSC spectrum of TYC 2627-638-1A with the main spectral features identified. Flux of broadband filters at the effective wavelengths of $B, V, R_{\mathrm{C}}$ are plotted with big (blue) stars; b) close-up of the $\mathrm{H} \alpha$ region from the spectrum taken at Ondřejov, which contains both components.

component and $\sim 60$ for the B component (measured around $6400 \AA$ ). With this échelle spectrograph a spectral range of 3700-7300 ̊ can be obtained in a single exposure. In the current work the 1.3 arcsec fibre with a resolving power $(\lambda / \Delta \lambda)$ of 46000 was used. The spectrum of the brighter star (TYC 2627638-1A) was obtained on 2007 July 17 and of the fainter one (TYC 2627-638-1B) on 2008 May 12. The exposure times were $2700 \mathrm{~s}$ and $3200 \mathrm{~s}$, respectively. During the observations the fibre was centred on the desired target, but due to the relatively large size of the fibre and seeing of 1.0-1.5 arcsec, some contamination from the other star cannot be avoided, but as shown in Sect. 3.3 this contamination is very small. On 2007 July 17 observations of a radial velocity standard, HD 161096, were obtained with the same setup as the target observations. The exposure time was set to $10 \mathrm{~s}$. All high-resolution spectra were reduced with the dedicated FIES reduction software FIEStool.

More high-resolution spectra of TYC 2627-638-1A were obtained using SOFIN high-resolution spectrograph, also at the Nordic Optical Telescope. These observations were carried out between 2009 August 26 and September 06 with the medium resolution camera and slit width of $65 \mu \mathrm{m}$, giving a resolving 
Table 3. The high-resolution spectroscopy of TYC 2627-638-1A with SOFIN at the Nordic Optical Telescope.

\begin{tabular}{lcrrrrr}
\hline \hline Date & $\begin{array}{c}\text { HJD } \\
2455000+\end{array}$ & $S / N$ & $R V$ & $\sigma R V$ & span & $\sigma$ span \\
& & \multicolumn{5}{c}{$/ \mathrm{s}]$} \\
\hline 2009 Aug. 26 & 70.4602 & 28 & -384 & 58 & 85 & 6 \\
2009 Aug. 27 & 71.4248 & 8 & 585 & 173 & 109 & 7 \\
2009 Aug. 28 & 72.4332 & 30 & 107 & 51 & 88 & 6 \\
2009 Aug. 29 & 72.5651 & 23 & 160 & 70 & 90 & 6 \\
2009 Aug. 29 & 73.4179 & 30 & 165 & 50 & 88 & 6 \\
2009 Aug. 31 & 75.4343 & 27 & 480 & 58 & 87 & 6 \\
2009 Sep. 1 & 76.4203 & 27 & 54 & 53 & 83 & 5 \\
2009 Sep. 2 & 77.4091 & 29 & 1 & 54 & 89 & 6 \\
2009 Sep. 3 & 77.5503 & 31 & -94 & 52 & 89 & 6 \\
2009 Sep. 3 & 78.4549 & 37 & 280 & 41 & 86 & 6 \\
2009 Sep. 4 & 79.4183 & 36 & -19 & 39 & 85 & 6 \\
2009 Sep. 5 & 79.5488 & 30 & 170 & 55 & 95 & 6 \\
2009 Sep. 5 & 80.4434 & 38 & 244 & 44 & 83 & 6 \\
2009 Sep. 6 & 81.4358 & 27 & 327 & 70 & 80 & 5 \\
\hline
\end{tabular}

power of 65000 . The exposure time of the individual exposures was typically $16 \mathrm{~min}$, yielding a signal-to-noise ratio at $6400 \AA$ between 8 and 38, typically better than 25 . These data were reduced and analysed with the 4A reduction package (Ilyin 2000). The main aim of these observations was to probe possible radial velocity $(R V)$ variations in TYC 2627-638-1A due to an unseen companion. The $R V$ was measured simultaneously for all the spectral orders without telluric lines, and with respect to an averaged spectrum (using orders 33-49, except the orders 36 and 38 ). The details of the observations and measured radial velocities together with their errors as well as bisector spans are given in Table 3.

For the bisector analysis the noise in the data was reduced with the LSD technique (see Semel 1989; Donati et al. 1997 for the algorithm). The technique simply assumes that profiles are similar in shape for all lines and only scale up in depth. Although this assumption is strictly valid only for optically thin lines, it is acceptable if very strong lines are excluded. The number of used lines (248) is limited by the échelle orders of the SOFIN spectrograph. For the LSD profiles using the blue and red part of the spectrum separately, 167 and 81 lines were used respectively.

\section{Results}

\subsection{Spectral classification: photometry, low-resolution spectrum}

The measured colour indices show that both TYC 2627-638$1 \mathrm{~A}$ and TYC 2627-638-1B are late-type stars, and their brightness suggests they are not too distant. The colour indices measured separately for TYC 2627-638-1A and TYC 2627-638-1B in its bright state (see below) are as follows (TYC 2627-638$1 \mathrm{~A} / \mathrm{TYC} 2627-638-1 \mathrm{~B}): B-V=0.61 / 0.72, V-R_{\mathrm{C}}=0.36 / 0.40$, $V-I_{\mathrm{C}}=0.72 / 0.78$. From the recent Kurucz tables (Castelli $\&$ Kurucz 2003) at solar composition and $\log g=4.0-4.5$ we find temperatures 5950-6000 K for TYC 2627-638-1A from the $B-V$ colour index, but about $150 \mathrm{~K}$ less from the red colour indices, probably due to spottedness. For TYC 2627-638-1B all colour indices point towards 5550-5600 K. For Main Sequence stars these temperatures correspond to early- (G1-2) and late-G spectral types. Note that the stars are close, at +20 galactic latitude (their distance is less than 250 pc, see Sect. 4.2), so the interstellar absorption does not considerably change the measured colours.
The low-resolution ALFOSC spectrum of TYC 2627-6381A clearly shows lines from the hydrogen Balmer series and metals like sodium, calcium and magnesium and a clearly visible G-band (see Fig. 2a). This implies that TYC 2627-638-1A is a cool star of the spectral type F5 or later. The G-band is slightly stronger than the $\mathrm{H} \gamma$-line, indicating spectral class of early $\mathrm{G}$, which is well in line with the photometric spectral classification of TYC 2627-638-1A. The average fluxes at the effective wavelengths of broadband colours $B, V, R_{\mathrm{C}}$ of TYC 2627-6381 A measured separately between 2007-2009, are also plotted in Fig. 2. The fluxes agree quite well. Note that the low-resolution spectrum is compared with the average photometry, but the longterm change of the bright component is small (see Sect. 3.4).

\subsection{Stellar parameters: high-resolution spectra}

Radial velocity, effective temperature, metallicity, Li abundance, $\log g$ and $v \sin i$ of both TYC 2627-638-1A and TYC 2627-638$1 \mathrm{~B}$ were measured from the high-resolution FIES spectra.

The spectra were analysed with the DAOSPEC analysis package of Stetson \& Pacino (2008), which semiautomatically derives radial velocity and equivalent widths. The line identifications for the automatic fitting and derivation of radial velocity were based on VALD linelists (Kupka et al. 1999; Piskunov et al. 1995). The fundamental parameters were then found following the procedure of Dall et al. (2005) employing the ATLAS9 port for Linux (Kurucz 1993; Sbordone et al. 2004), interpolating in the grid of Kurucz model atmospheres. The abundance analysis and parameter fitting was done relative to the Sun, using the high quality HARPS solar spectrum also used by Dall et al. (2006) because no solar spectrum is available for FIES. Due to the larger rotational broadening in TYC 2627-638-1A only four reliable Fe II lines could be found, which is the main contributor to the uncertainty of the parameters for TYC 2627-638-1A.

For TYC 2627-638-1A the best fit was obtained with $T_{\text {eff }}=$ $5900 \pm 100 \mathrm{~K}, \log g=4.0 \pm 0.3$, microturbulent velocity $\xi_{t}=$ $2.0 \mathrm{~km} \mathrm{~s}^{-1}$ and $[\mathrm{Fe} / \mathrm{H}]=0.0 \pm 0.2$, and for TYC 2627-638-1B using $T_{\text {eff }}=5800 \pm 80 \mathrm{~K}, \log g=4.60 \pm 0.13, \xi_{t}=1.6 \mathrm{~km} \mathrm{~s}^{-1}$ and $[\mathrm{Fe} / \mathrm{H}]=0.02 \pm 0.12$. For Main Sequence stars these effective temperatures would indicate spectral types approximately G0-1 and G2 for TYC 2627-638-1A and TYC 2627-638-1B, respectively.

A region in the red part of the spectrum spanning wavelengths 6374-6454 $\AA$ was chosen for investigating the radial velocity and $v \sin i$ because it contains several metal lines, mainly $\mathrm{Fe}$ and $\mathrm{Ca}$, and it also has a good signal-to-noise ratio. The radial velocity of the two stars was measured with the fxcor routine in IRAF. The standard HD 161096 has a published velocity of $12.53 \mathrm{~km} \mathrm{~s}^{-1}$ (Famaey et al. 2005). The heliocentric radial velocity for TYC 2627-638-1A and for TYC 2627-638-1B are $-23.0 \pm 4.6 \mathrm{~km} \mathrm{~s}^{-1}$ and $-20.1 \pm 4.4 \mathrm{~km} \mathrm{~s}^{-1}$, which agree well with each other within $1 \sigma$.

The $v \sin i$ measures of the two stars were determined by modelling the 6374-6454 A spectral region with different values of $v \sin i$, but fixing the effective temperature to the above mentioned values. Also, solar abundance and $\log g=4.5$ were used in the modelling. The list of the atomic lines in the wavelength region was again obtained from VALD, and the stellar atmosphere models from Kurucz were used in the modelling. The synthetic spectra for different $v \sin i$ were calculated with the SLOC spectral synthesis code (Berdyugina 1991). The best correspondence between the observations of TYC 2627-638-1A 
Table 4. Properties of TYC 2627-638-1A and TYC 2627-638-1B as determined from the high-resolution FIES spectra.

\begin{tabular}{lll}
\hline \hline Parameter & TYC 2627-638-1A & TYC 2627-638-1B \\
\hline$T_{\text {eff }}$ & $5900 \pm 100 \mathrm{~K}$ & $5800 \pm 80 \mathrm{~K}$ \\
$\log g$ & $4.0 \pm 0.3$ & $4.6 \pm 0.13$ \\
metallicity $[\mathrm{Fe} / \mathrm{H}]$ & $0.0 \pm 0.2$ & $0.02 \pm 0.12$ \\
$\mathrm{Li} \mathrm{EW}$ & $182 \mathrm{~m} \AA$ & $192 \mathrm{~m} \AA$ \\
$v \sin i$ & $18 \pm 2 \mathrm{~km} \mathrm{~s}^{-1}$ & $4 \pm 1 \mathrm{~km} \mathrm{~s}^{-1}$ \\
heliocentric $R V$ & $-23.0 \pm 4.6 \mathrm{~km} \mathrm{~s}^{-1}$ & $-20.1 \pm 4.4 \mathrm{~km} \mathrm{~s}^{-1}$ \\
\hline
\end{tabular}

and the synthetic spectrum was obtained with $v \sin i=18 \mathrm{~km} \mathrm{~s}^{-1}$, and for TYC 2627-638-1B with $v \sin i=4 \mathrm{~km} \mathrm{~s}^{-1}$.

The obtained stellar parameters for TYC 2627-638-1A and TYC 2627-638-1B are summarised in Table 4.

\subsubsection{Age estimates of the system}

The Li abundance was measured with the Li 6708 blend. The obtained equivalent widths (EW) for TYC 2627-638-1A and TYC 2627-638-1B are $182 \mathrm{~m} \AA$ and $192 \mathrm{~m} \AA$, respectively. This indicates that both stars are young and have an age younger than that of the Pleiades. According to Mentuch et al. (2008) early G-type stars in the $\beta$ Pic moving group and in the Tucanae-Horologium association have similar Li EW to that of TYC 2627-638-1A and TYC 2627-638-1B, and from this we may estimate an age of about $25 \mathrm{Myr}$ with an uncertainty of at least 10 Myr for our objects.

From the high-resolution spectra calcium S-index for TYC 2627-638-1A and TYC 2627-638-1B were measured with the $\mathrm{H}$ line, giving $\mathrm{S}(\mathrm{A})=0.54 \pm 0.06$ and $\mathrm{S}(\mathrm{B})=$ $0.61 \pm 0.09$. Transforming these values to $R_{H K}^{\prime}$ indices with the theoretical $B-V$ values from Siess et al. (2000) we get $\log R_{H K}^{\prime}(\mathrm{A})=-4.13_{-0.06}^{+0.05}$ and $\log R_{H K}^{\prime}(\mathrm{B})=-4.10_{-0.08}^{+0.03}$, taking into account the measurement errors. Thus the $\mathrm{S}$ index values can be regarded as the same for the two components. Another source of uncertainty are the possible systematics due to the lack of accurate transformation to the Mount Wilson type S index, which we suppose to be at most $10 \%$ (for a discussion of this problem see e.g. Schröder et al. 2009). With all the different types of errors the $\log R_{H K}^{\prime}$ value should be between -4.0 and -4.25 , indicating an age of 10-100 Myr (Mamajek \& Hillenbrand 2008) with the most possible value of about $30 \mathrm{Myr}$, in good agreement with the $\mathrm{Li}$ age.

From the $\log R_{H K}^{\prime}$ value of about -4.1 and equation A2 from Mamajek \& Hillenbrand (2008), we get $L_{X} \sim 3 \times 10^{30} \mathrm{erg} / \mathrm{s}$. The estimated value at the distance of $230 \mathrm{pc}$ (Sect. 4.2), would be at the detection limit of ROSAT, which indeed has no source at the position of TYC 2627-638-1.

The space velocity of the system $U=-25.9 \pm 3.7, V=$ $-7.3 \pm 3.5, W=-9.3 \pm 2.5 \mathrm{~km} \mathrm{~s}^{-1}$ from the measured average radial velocity of $-21.5 \mathrm{~km} \mathrm{~s}^{-1}, 230 \mathrm{pc}$ distance (Sect. 4.2) and published proper motion (UCAC3, Zacharias et al. 2010) agrees well with the velocity of the young disc population of the Galaxy (see Moór et al. 2006, Fig. 1.), which further strengthens the young age of the stars.

\subsection{Signatures of magnetic activity}

The high-resolution FIES spectra of TYC 2627-638-1A and TYC 2627-638-1B were also used for investigating activity

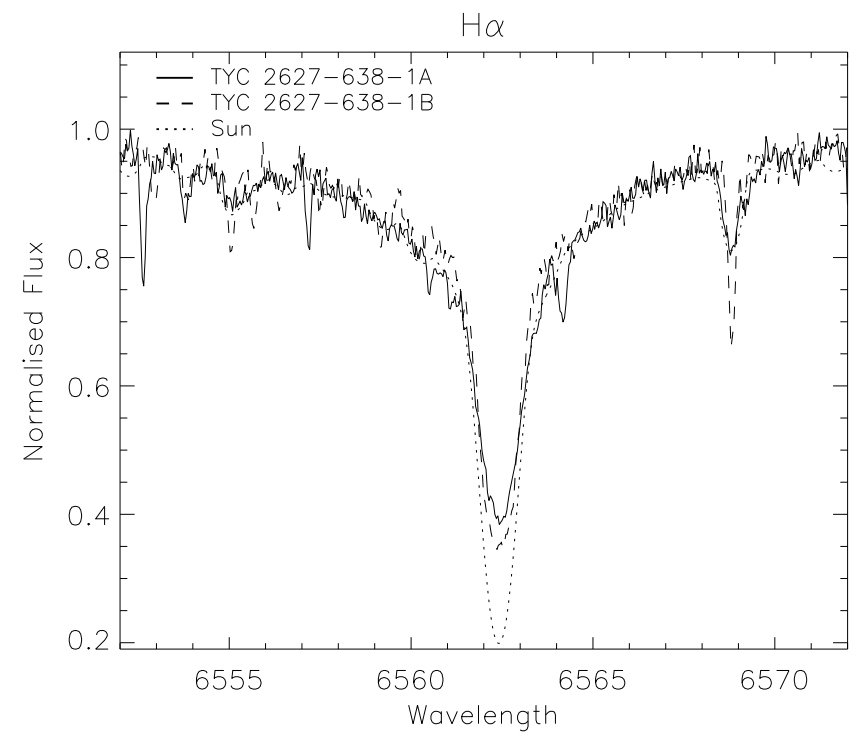

Fig. 3. H $\alpha$ lines from TYC 2627-638-1A and TYC 2627-638-1B plotted together with the solar spectrum that has been broadened to $v \sin i=$ $18 \mathrm{~km} \mathrm{~s}^{-1}$. TYC 2627-638-1B and solar spectra have been moved to the same reference frame as TYC 2627-638-1A spectrum.

signatures. For this purpose the $\mathrm{H} \alpha$ and $\mathrm{Ca}$ II $\mathrm{H} \& \mathrm{~K}$ lines were studied in detail.

Figure 3 shows the $\mathrm{H} \alpha$ line profiles of TYC 2627-638-1A and TYC 2627-638-1B. Neither of the stars shows clear emission features in the $\mathrm{H} \alpha$ line, but in both of them the line is shallower than in the solar case and could be filled-in with chromospheric emission.

$\mathrm{Ca}$ II H\&K lines are very good indicators of stellar activity. In Fig. 4 the region containing these lines is shown for both stars. In Fig. 4a the whole region is shown with TYC 2627-638-1A spectrum plotted above the TYC 2627-638-1B spectrum. Both stars show clear emission cores in $\mathrm{Ca}$ II $\mathrm{H} \& \mathrm{~K}$ lines. Figure $4 \mathrm{~b}$ shows the $\mathrm{Ca}$ II $\mathrm{H}$ line spectrum of both stars plotted on top of each other, and Fig. 4c the $\mathrm{Ca}$ II $\mathrm{H}$ line core region in a similar way. The spectra are very similar, except that the absorption lines at the wings of the line are clearly more narrow in TYC 2627-638$1 \mathrm{~B}$ than in TYC 2627-638-1A, which is in line with the $v \sin i$ measurements presented earlier.

One has to remember though, that the fibre aperture of FIES is 1.3 arcsec and the seeing during the observations was 1.0-1.5 arcsec, thus the TYC 2627-638-1B spectrum can have contamination from the brighter companion, which is 1.8 arcsec away from it. And as TYC 2627-638-1B is the fainter and redder of the two, the contamination would be more noticeable in the blue part of the spectrum. Still, the position of the emission cores in each star corresponds to its radial velocity, so any contamination of the spectra due to the proximity on the sky is negligible. Therefore both stars have to be treated as magnetically active.

\subsection{Photometric variability}

The bulk of the photometric measurements was made with the $60-\mathrm{cm}$ telescope in Budapest and only in $B V I_{\mathrm{C}}$ colours. Thus these colours were chosen for the following analysis (see data plotted in Fig. 5), which contains the light of both TYC 2627638-1A and TYC 2627-638-1B. The mean light of the system is continuously decreasing in all colours during the observations between 2006-early 2009, but started to slowly increase from 

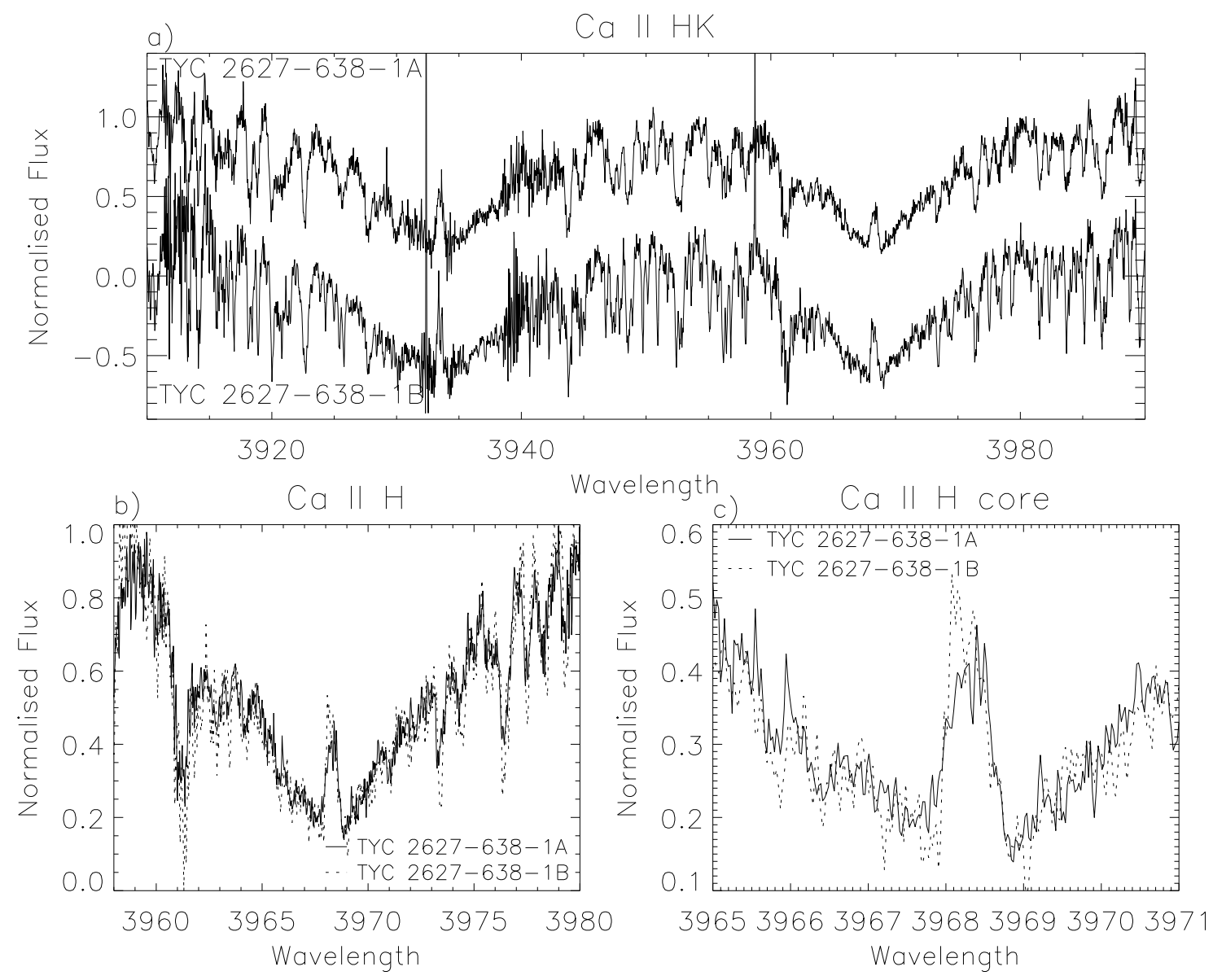

Fig. 4. Ca II H\&K lines from TYC 2627-638-1A and TYC 2627-638-1B plotted together: a) the whole spectral region; b) the Ca II H line in detail; c) the $\mathrm{Ca}$ II $\mathrm{H}$ line core. In b) and c) TYC 2627-638-1A is plotted with a solid line and TYC 2627-638-1B with a dotted line. The TYC 2627-638-1B spectrum has been moved to the same reference frame as TYC 2627-638-1A spectrum.

July 2009 onwards. Variations on a yearly timescale are also present.

The standard deviation of the separate average magnitudes of TYC 2627-638-1A and TYC 2627-638-1B given in Table 2 compared to those for the comparison star $\mathrm{C} 1$ in Table 1 hint at some low-amplitude variation for TYC 2627-638-1A and high-amplitude changes for TYC 2627-638-1B (C1 is about 1.2 mag. fainter than TYC 2627-638-1A, and similar in brightness to TYC 2627-638-1B). TYC 2627-638-1B became fainter by $0.3 \mathrm{mag}$. in $V$ colour between mid-2008 and early-2009, and in the same time the star became redder by about $0.1,0.04$ and 0.07 mag. in $B-V, V-R_{\mathrm{C}}$ and $V-I_{\mathrm{C}}$ colour indices, respectively. In September 2009 the brightness of TYC 2627-638-1B jumped back by $0.35 \mathrm{mag}$. to the previous value, and in October 2009 was again in its low state. Figure 6 shows the long-term variability of TYC 2627-638-1B, where magnitudes of TYC 2627638-1A and the check star from the same frames are also plotted for comparison. The typical errors of the colour index data for TYC 2627-638-1B is quite high; the reliability of the change is demonstrated in the next section.

\subsubsection{Periodic changes}

We checked the dataset for periods using Fourier analysis on the $V$ dataset in which the magnitudes contain both TYC 2627-638$1 \mathrm{~A}$ and TYC 2627-638-1B together. The result is displayed in Fig. 7. First we pre-whitened the data with the long-term trend (a long period plus its 2 harmonics) and checked again if there was any signature of periodicity in a rotational timescale. Two close but separate and real periods near 3.5 and 3.7 days were found, and a weak signal of about 63 days with about $0.006 \mathrm{mag}$. full amplitude. The probable origin of these periods is discussed in the two paragraphs of this section.

Then we further checked the two close periods near 3.5 and 3.7 days. Because the length of the rotational modulation could change due to differential rotation after some time, we split the dataset into three parts. The search for rotational period(s) was repeated separately for the observations of the three seasons 2006, 2007 and 2008-early 2009. At this stage we used a periodfinding algorithm based on the Lafler-Kinman method (Clarke 2002), which is useful for finding periods of a non-sinusoidal shape, which is often characteristic of active star light curves. The result is plotted in Fig. 8.

The period search gave interesting results. In 2006 the 3.5 days period has a much higher amplitude than the 3.7 days signal, which is quite weak. By 2007 the amplitudes of the two periods became similar, which can be due to changes in the spot positions and/or sizes. The derived periods for 2006 and 2007 are consistent for $B V I_{\mathrm{C}}$ data, i.e., are well within the 0.01-0.02 uncertainty of the individual determinations. The final rotational periods from the three colour datasets are as follows: for all the data together: $P_{1}=3.496 d$; for $2006 P_{1}=3.496 d$ and $P_{2}=3.70 d$, and for $2007 P_{1}=3.500 d$ and $P_{2}=3.68 d$. With these periods we plotted the light and the colour index curves 


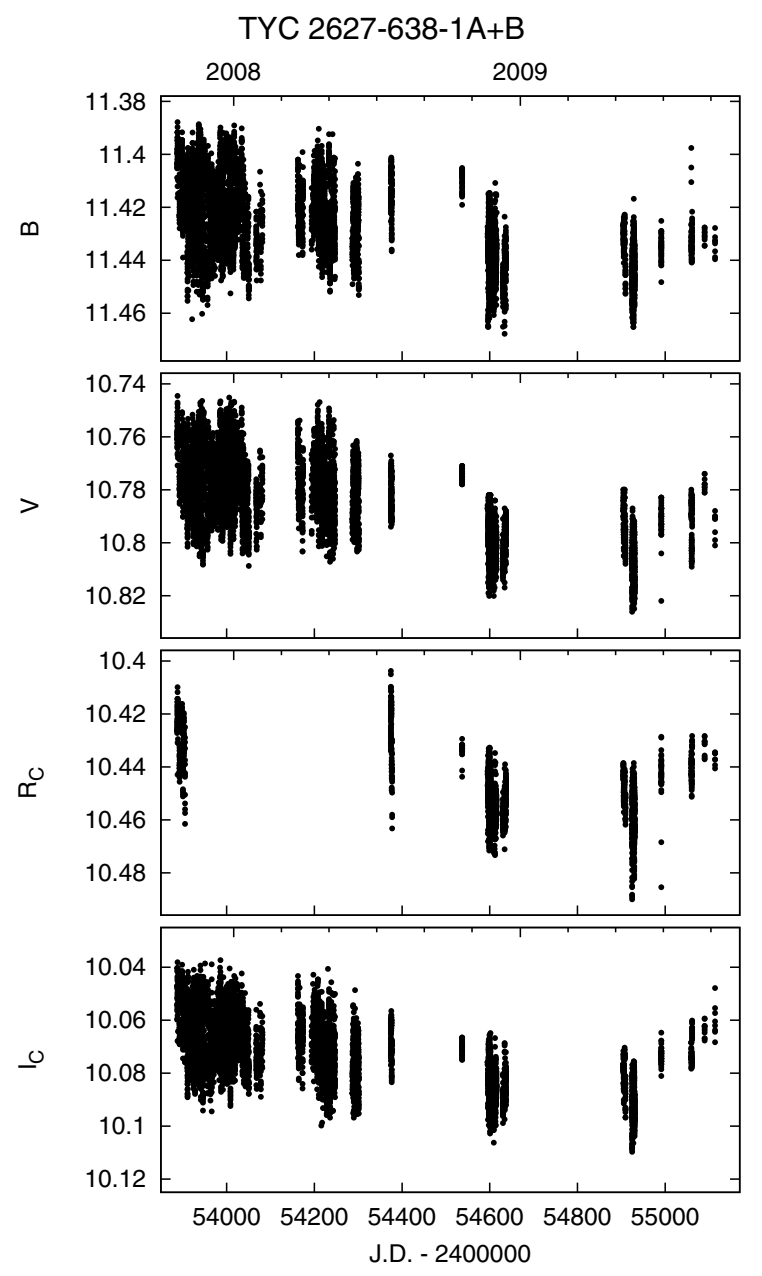

Fig. 5. From top to bottom: $B, V, R_{\mathrm{C}}, I_{\mathrm{C}}$ data.

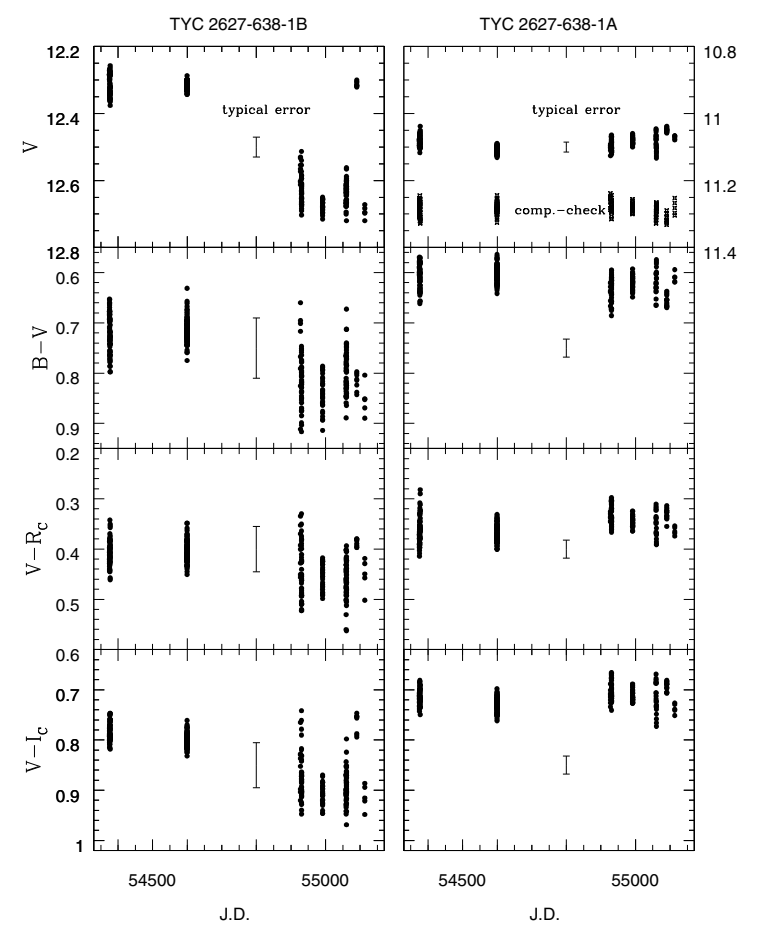

Fig. 6. $V$ magnitudes and colours of TYC 2627-638-1A and TYC 2627638-1B on 11 excellent nights between 2007-2009. The seeing enabled us to separate the two stars with PSF photometry. The companion star shows strong variability on a longer timescale. Comparison-check magnitudes are plotted in the upper right panel, shifted by +1.6 mag.
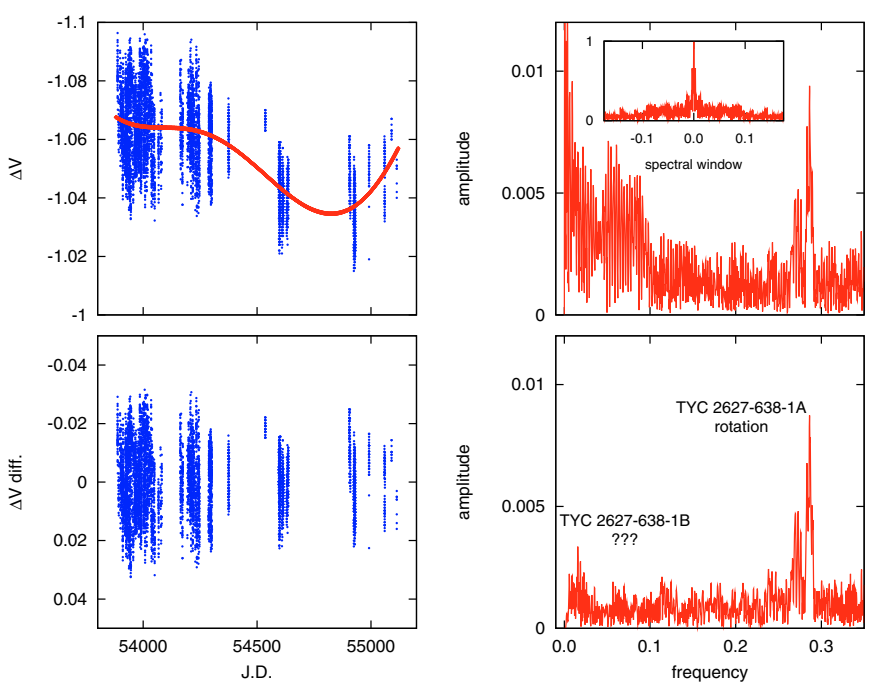

Fig. 7. Result of the Fourier analysis of the $V$ colour data. The upper two panels show the dataset and the amplitude spectrum with the spectral window in the insert. Below are plotted the data set pre-whitened with the long-term trend and its amplitude spectrum, with two signatures of periodicities.

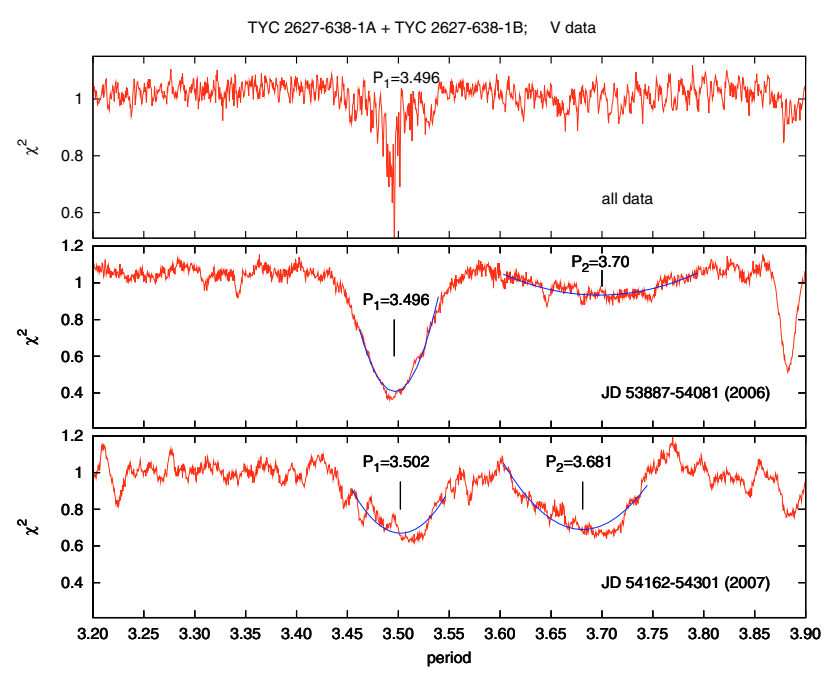

Fig. 8. Result of the period search in $V$ colour. From top to bottom the results for all data and for the 2006 and 2007 datasets are plotted. Details are given in the text.

in Fig. 9. Amplitude differences between the three colour light curves with both periods in 2006 and 2007 are small or none (Fig. 9). The bottom panels show the behaviour of the colour indices. Only the $V-I_{C}$ colour index in 2006 and maybe in 2007 belonging to the 3.5 days period show a small rotational modulation.

In 2008-2009 the dataset is more sparse and the number of data is less than in the previous years, so the period search is uncertain and no rotational period is directly found. Plotting all the data from 2008-2009 with the resulting periods from 20062007, we get just scatter plots.

TYC 2627-638-1A However, when we take those seven nights from 2008 May to 2009 September, when the stars could have been measured separately, we find that the main period 

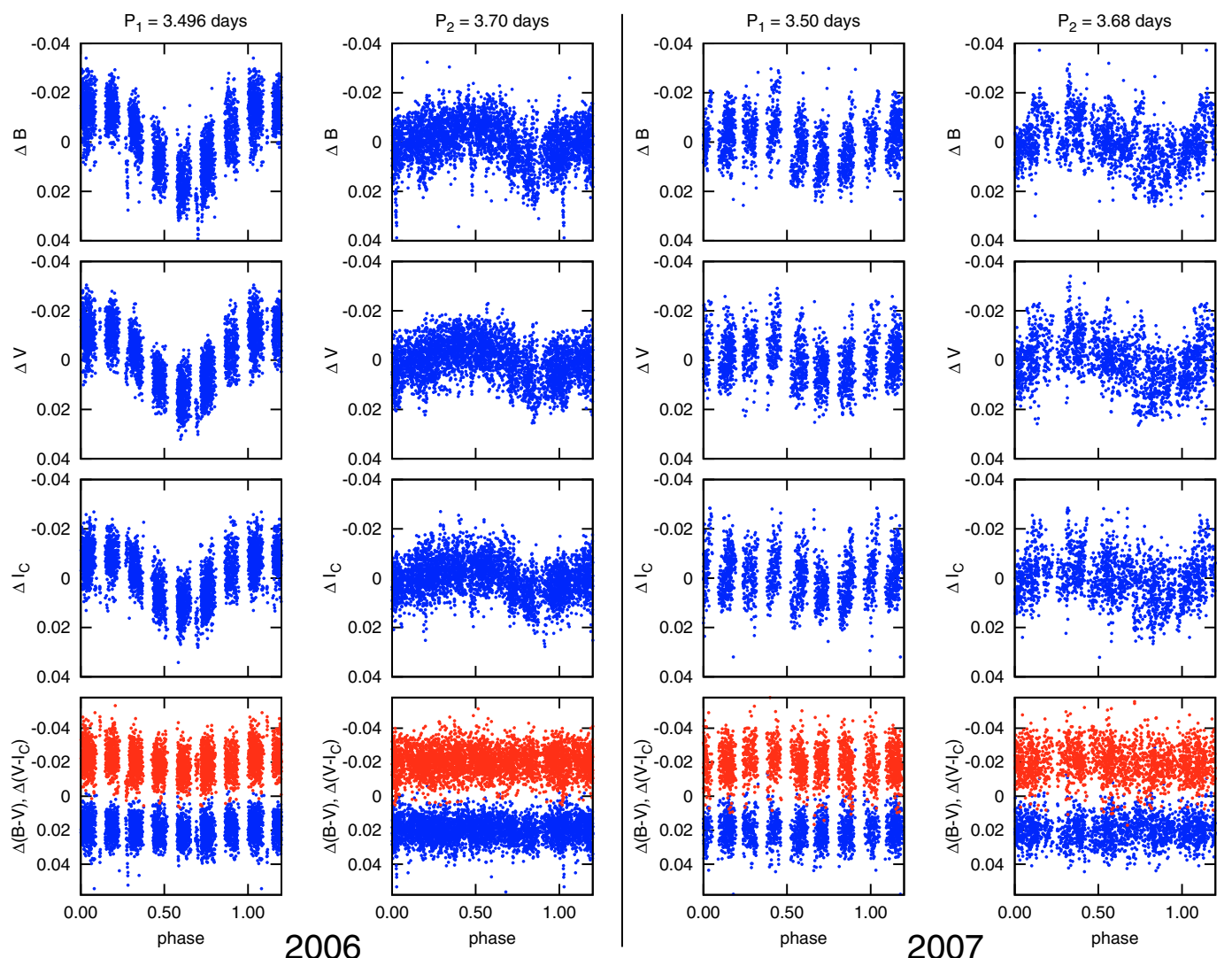

Fig. 9. Light and colour index curves from the $V$ dataset (the two components together) folded with $P_{1}$ and $P_{2}$, the data are pre-whitened with the other period. Left two panels: 2006 (JD 53887-54011), right two panels: 2007 (JD 54162-54301) The bottom panels shows the colour index curves, in each panel up is $B-V$, down is $V-I_{\mathrm{C}}$, the data are shifted by \pm 0.02 mag for clarity.

$P_{1}=3.496 d$ from all the data applies well to the small amplitude light variation of TYC 2627-638-1A. Removing this variation we find that $P_{2}=3.70 \mathrm{~d}$ also fits the pre-whitened data. The light curve of TYC 2627-638-1A with these two periods are plotted in Fig. 10 together with the $V-I_{\mathrm{C}}$ colour indices. Folded with the main period $P_{1}=3.496 \mathrm{~d}$, the $V-I_{\mathrm{C}}$ colour index curve runs parallel with the $V$ lightcurve. The two distinct periods present in the photometric data thus can be interpreted with two active regions at different latitudes on TYC 2627-638-1A, and the surface differential rotation cause them to rotate with different periods. The light curves in Figs. 9 and 10 suggest a long-term stability of the variations and thus the activity on time-scales from several months to perhaps years. The data from 2009 October do not fit the folded light curve of TYC 2627-638-1A well, due to a probable change in the spot distribution.

TYC 2627-638-1B The high amplitude variability in (cf. Fig. 6, left) changed monthly in 2009 August-October, but remained at about the same brightness within a week in all cases. The observations suggest a tentative period of $59.5 \pm 1.5$ days in the 0.35 mag change in $V$ colour, accompanied by parallel colour index change, plotted in Fig. 11. The Fourier analysis of the whole dataset revealed a weak signal with about 0.006 mag. full amplitude, near 63 days (cf. Fig.7). This suggests that the similar periodicity we find from the separately measured data for TYC 2627-638-1B is probably present all the time. Note that the 0.35 mag. amplitude of TYC 2627-638-1B is reduced to about $0.008 \mathrm{mag}$. when both stars are measured together by the much brighter TYC 2627-638-1A (cf. Table 2), and this is the reason for the low amplitude of the 63 days period found in the

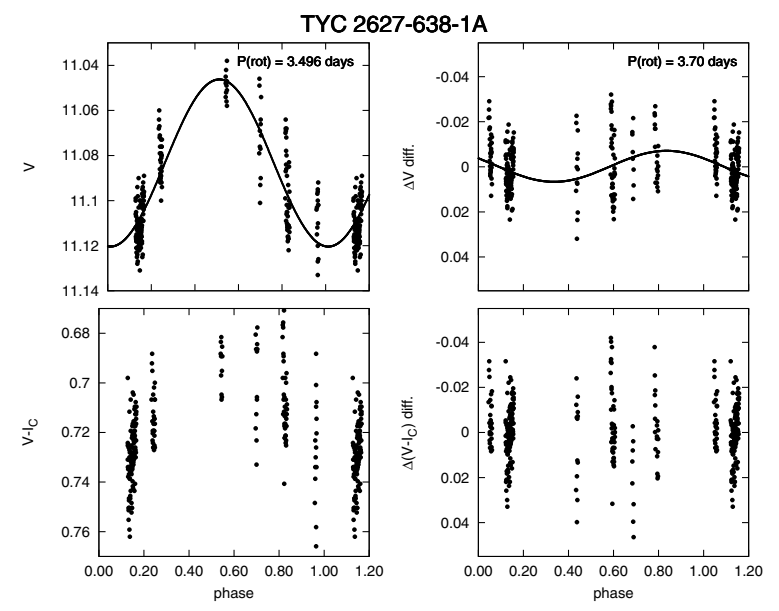

Fig. 10. Light curve of TYC 2627-638-1A, measured separately between 2008 May - 2009 September on seven nights. Upper panels, left: data folded with the main period $P_{1}=3.496 \mathrm{~d}$, right: the data pre-whitened with $P_{1}$ and folded with $P_{2}=3.70 \mathrm{~d}$. Bottom panels: the corresponding $V-I_{\mathrm{C}}$ colour indices.

Fourier analysis (Fig. 7). The origin of this variability is unclear. Colour-brightness diagrams are plotted both for TYC 2627-638$1 \mathrm{~A}$ and TYC 2627-638-1B in Fig. 12. No relation is found between $V$ and $B-V$ and only a marginal one between $V$ and $V-I_{\mathrm{C}}$ in TYC 2627-638-1A. The latter is most likely due to spottedness. However, strong trends are seen between the brightness and colours of TYC 2627-638-1B with about the slope of the general reddening function. 

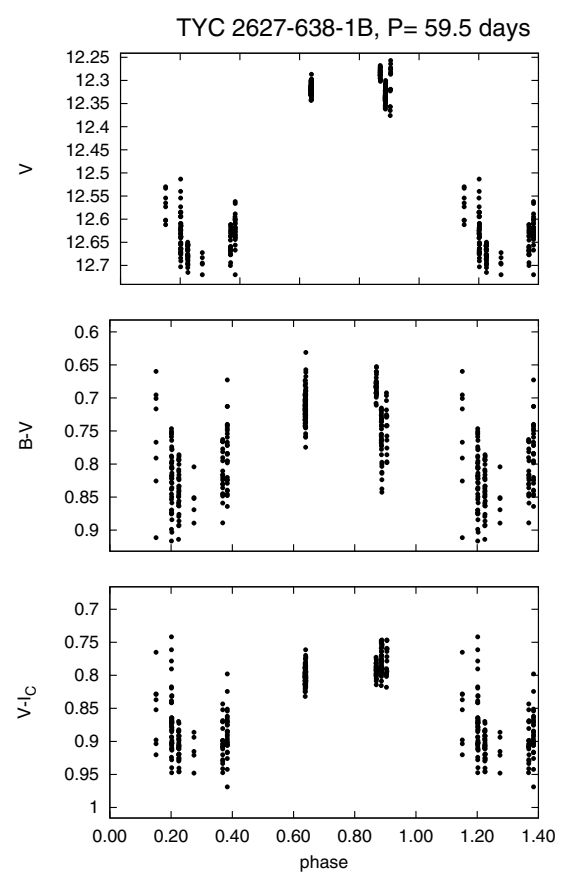

Fig. 11. Light variability of TYC 2627-638-1B during two full years phased with a quasiperiod of 59.5 days, using separately measured data from 11 nights.
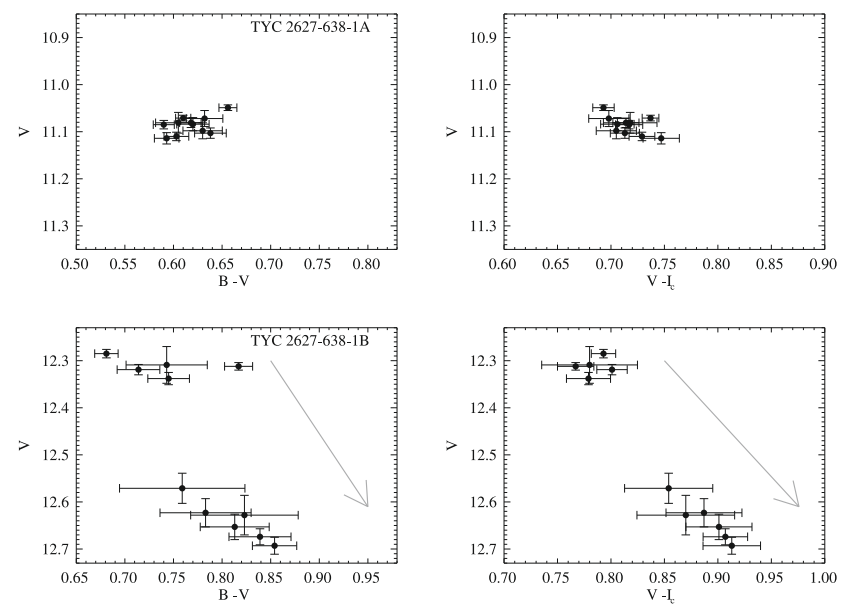

Fig. 12. Colour-magnitude diagrams for TYC 2627-638-1A and TYC 2627-638-1B drawn from the nightly averaged magnitudes measured separately on 11 nights (cf. Figs. 10 and 11). The reddening line (corresponding to $E(B-V)=0.1$ is plotted in the bottom panels. See details in the text.

\subsection{Other unseen companions of TYC 2627-638-1A}

The radial velocities presented in Table 3 suggest an unseen companion of TYC 2627-638-1A orbiting with a period of 1.59 \pm 0.02 days and with a total velocity amplitude of $402 \pm 43 \mathrm{~m} / \mathrm{s}$. Figure 13 shows the radial velocity data plotted with this period and the corresponding bisector span for each datapoint. There is no relation between the phased velocity and bisector span values suggesting that the velocity variation is indeed of orbital origin. The Fourier analysis of the radial velocity data, excluding the two deviating datapoints, yields a period of $1.58 \pm 0.02$ days, with a total amplitude of $436 \mathrm{~m} / \mathrm{s}$, i.e., we get the same values as from the orbital solution within $1 \sigma$. The short time base and the low number of measurements do not allow us to derive a well-determined orbital period. The value we find (1.59 days) is
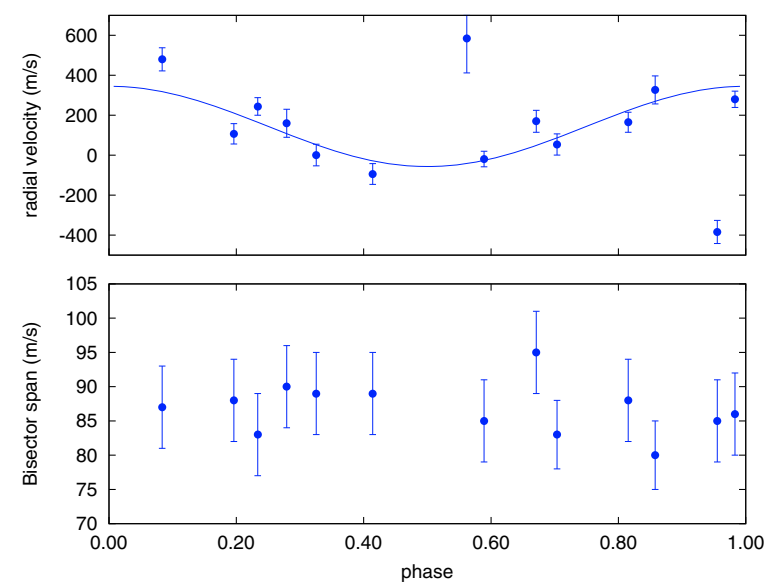

Fig. 13. Radial velocity measurements of TYC 2627-638-1A folded with a 1.59 days period and the corresponding bisector spans on the same velocity scale.

unrelated to the rotational period of the star (3.5 and 3.7 days) that appears by rotating starspots. Thus we argue that the spots do not mimic a radial velocity change. The few hundred meters/sec velocity amplitude points toward a substellar companion, but without information on the orientation of the orbital plane no good estimate can be given as to its mass. This companion is named as TYC 2627-638-1Aa.

However, because it looks quite certain that the low amplitude 3.5 days light variability of TYC 2627-638-1A originates from starspots, it means that its inclination cannot be very close to zero (i.e., polar view). Supposing an inclination of $\geq 15^{\circ}$ and that the rotational axis is perpendicular to the orbital plane, the mass of the substellar component TYC 2627-638-1Aa is $\leq 5$ Jupiter masses, orbiting the $\approx 1.25 M_{\odot}$ star (see Sect. 4.2).

We plot the 14 LSD profiles of the SOFIN spectra (2009 August-September) and one LSD profile of the FIES spectrum (2007 July) of TYC 2627-638-1A in Fig. 14 separately for the blue and red part of the spectra. Because the $S / N$ of the spectra was very low (see Table 3) and it was only possible to use a limited number of lines, even LSD was not able to remove all the noise, as can be seen from the profiles. The shapes of the SOFIN spectra match each other well, and give very similar bisectors (Fig. 13), besides they show a definite bump at about $+20 \mathrm{~km} / \mathrm{s}$. The FIES spectrum (above the SOFIN spectra) shows the same characteristics. This bump probably originates from yet another star within the tiny area of less than 2 arcsec in diameter. Because the bump is more pronounced in the red, the star should be of later spectral type. The position of the bump did not change for more than two years which suggests a background or foreground object not physically related to TYC 2627638-1A. The chance of such an alignment is quite low, but as this component is faint, the probability is higher than the $0.01 \%$ estimated for TYC 2627-638-1B. If this star is in the foreground or background with respect to TYC 2627-638-1A, then due to the proper motion the relative position of the two stars may change during decades. Checking the POSS and HST images made about 60 years and again more than 10 years ago, the image of TYC 2627-638-1A is always spherical, there seems to be no difference between the old and recent images.

Another possibility to explain the bump in the LSD profiles is that TYC 2627-638-1A is itself a spectroscopic binary, and we unluckily observed it about the same phase in 2007 and 2009 during 11 nights. This would mean a long period as well, but 


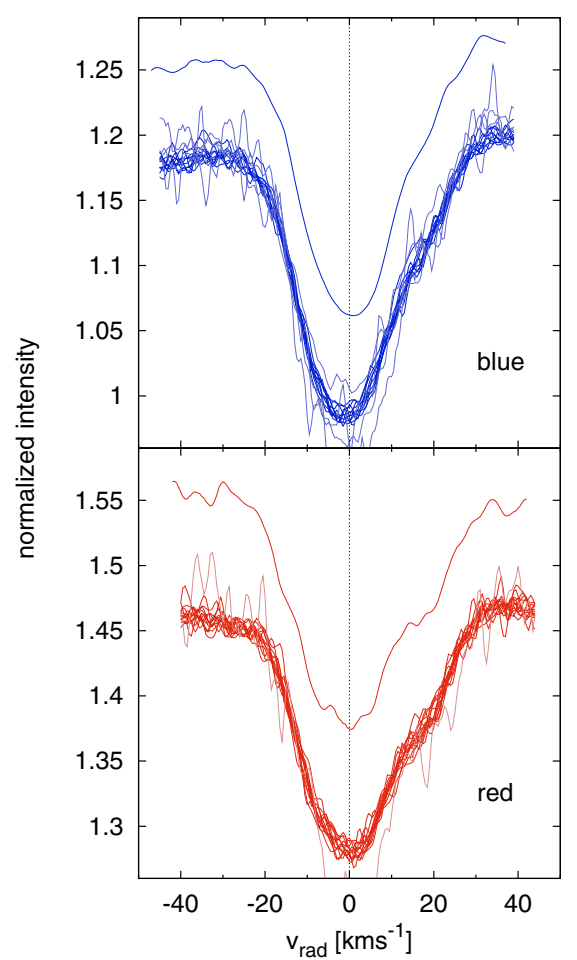

Fig. 14. Blue and red parts of the LSD profiles of the 14 SOFIN spectra and the FIES spectrum (above them) of TYC 2627-638-1A. A bump is seen around $+20 \mathrm{~km} \mathrm{~s}^{-1}$ and looks stronger in the red part.

spectroscopic binaries usually have not too long periods. Taking into account the bump in the blue and red LSD profiles, this star, which we now name TYC 2627-638-1C, should be much fainter (less than $10 \%$ flux, probably only a few \%) than TYC $2627-$ 638-1A. The spectroscopic binary scenario of TYC 2627-638$1 \mathrm{~A}$ with a later type star would reconcile the small difference between the spectral types found from photometry and from spectroscopy. However, the probability that we observed the binary about the same orbital phase in 2007 and 2009 is not very high. Because it is not possible to derive the spectral type of TYC 2627-638-1C, and knowing that it is much fainter, we neglect its additional light to the measurements of TYC 2627-638$1 \mathrm{~A}$.

\section{Discussion}

\subsection{Rotational modulations}

We find a photometric variability with periods near 3.5 and 3.7 days, possibly due to starspots, situated on TYC 2627-6381A. The activity is suggested by the Ca II H\&K emission cores belonging to this G0 star. The radius of TYC 2627-638-1A is about 1.25 solar radii from the HRD of $25 \mathrm{Myr}$ (Siess et al. 2000). Using its 3.5-3.7 days rotational periods this radius gives about $18 \mathrm{~km} \mathrm{~s}^{-1}$ rotational velocity, well comparable with the spectroscopically derived $v \sin i=18 \pm 2 \mathrm{~km} \mathrm{~s}^{-1}$. The fast rotation of at least one component in a wide binary system also suggests a young age.

The fainter visual companion TYC 2627-638-1B shows high amplitude light variation on a timescale of about 60 days, which one might think is of rotational origin based on the activity indicator Ca II H\&K emission cores. The young age of this star however, contradicts to the rotational explanation of the slow variation as shown in Mamajek \& Hillenbrand (2008, their Fig. 9).

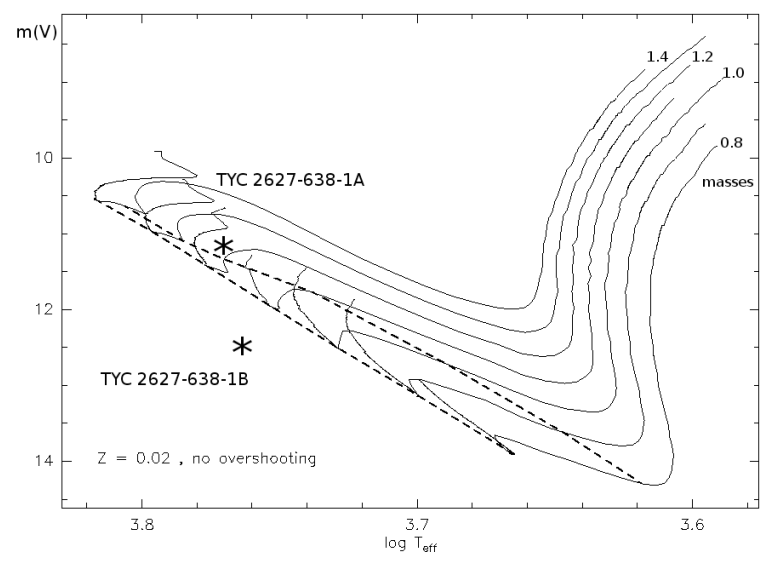

Fig. 15. Position of the stars on the HRD with a distance modulus of 7.01 derived for the G0 component TYC 2627-638-1A. The visual companion TYC 2627-638-1B is too faint for its derived temperature.

Also, the $\sim 60$ days periodicity is too long even for the low $v \sin i=4 \pm 1 \mathrm{~km} \mathrm{~s}^{-1}$. However, the colour-magnitude relations plotted on Fig. 12 suggest that the light variation on this long timescale may (at least partly) originate from some moving dust around the star.

\subsection{The visible components: single stars or a binary?}

The radial and space velocities, the similar spectral types, lithium abundances and $\mathrm{Ca} \mathrm{S}$ indices, and hence age, make it likely that the two stars of the visual binary TYC 2627-638-1A and TYC 2627-638-1B form a bound system of common origin. At a galactic latitude of $20^{\circ}$ the probability that TYC 2627-638$1 \mathrm{~B}$ is by chance aligned with TYC $2627-638-1$ A within $1.84^{\prime \prime}$ is less than $0.01 \%$ (cf. Sect. 2.1). From this it is clear that these two stars are almost certainly physical components. Because the radial velocities of TYC 2627-638-1A and TYC 2627-638-1B are within $3-4 \mathrm{~km} \mathrm{~s}^{-1}$, the by-chance alignment probability is reduced by another order of magnitude. Because both stars show strong Li this probability is reduced by at least another order of magnitude. Using a 230 pc distance and knowing the visible separation of 1.84 arcsec, the true separation of the two visible components is about $450 \mathrm{AU}$, which is typical of a wide binary system. Simple calculations suggest that the orbital period of this system is of the order of 6000-yr and the orbital velocity is of the order of $3 \mathrm{~km} \mathrm{~s}^{-1}$. Therefore there is no way to measure the variation in the radial velocity with this period and orbital motion. From the archives only on the POSS red and infrared images are two stars somewhat separately visible in a very similar position angle to the one they are at present.

Taking into account the interstellar extinction from the Schlegel maps (Schlegel et al. 1998) we get a distance of $218 \mathrm{pc}$ for the G0 star TYC 2627-638-1A with the total absorption of $A_{V}=0.32$ and $252 \mathrm{pc}$ supposing zero absorption $(M(V)=4.09$, Siess et al. 2000). Only a few parsecs error result from the uncertainty of the absorption value.

A pre-Main Sequence Hertzsprung-Russell Diagram (HRD) is plotted with the ZAMS and an isochrone of 25 Myr in Fig. 15 for a distance modulus of 7.01 using $m(V)=11.1, M(V)=4.09$, G0 sp. type (Siess et al. 2000). The plot reveals that the positions of the G0 star TYC 2627-638-1A is slightly above the MS near mass of 1.2 close the $25 \mathrm{Myr}$ isochrone. The position of the G2 star TYC 2627-638-1B shows that it is too faint for its derived temperature. If the visual binary forms a bound system as 


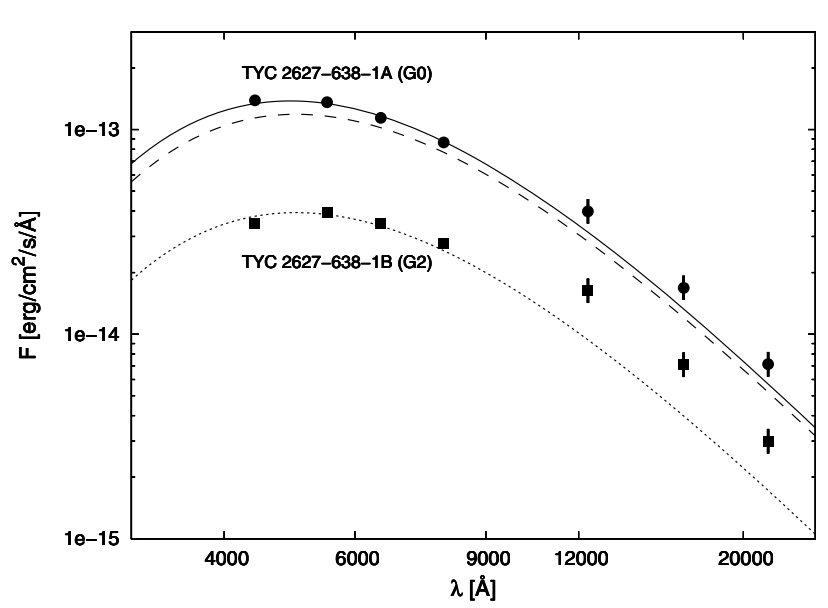

Fig. 16. Mean $B V(R I)_{\mathrm{C}}$ and $J H K s$ fluxes measured for the two visible components TYC 2627-638-1A and TYC 2627-638-1B, the errorbars on the JHKs fluxes represent both the possible systematic and quality uncertainties. Blackbody curves are drawn for $230 \mathrm{pc}$ distance with solid and dashed lines for TYC 2627-638-1A and TYC 2627-638-1B, respectively. A blackbody curve at $400 \mathrm{pc}$ distance is plotted with a dotted line for TYC 2627-638-1B. Discussion is in the text.

suggested above, something blocks the light of the fainter star. The origin could be a dust cloud around the star whose characteristics are not known.

We plotted the spectral energy distribution (SED) consisting of the broadband $B V(R I)_{\mathrm{C}}$ and the $J H K s$ fluxes for the G0 star TYC 2627-638-1A and for the visual companion G2 star TYC 2627-638-1B in Fig. 16 from the values from Table 2. In $B V(R I)_{\mathrm{C}}$, the low amplitude rotational modulation of the bright component causes negligible modulation in the fluxes, whereas the higher amplitude changes of the fainter component result in about the same amount of flux changes as the estimated error in $J H K s$, i.e., the light variability does not change the overall picture the figure suggests. Near-infrared excess is seen both for TYC 2627-638-1A and TYC 2627-638-1B; similar excess is found from the Tycho- 2 and 2MASS fluxes, which were measured containing both stars.

The solid line on Fig. 16 represents a blackbody curve for $T_{\text {eff }}=5900 \mathrm{~K}$ (i.e., for the derived temperature of the G0 star TYC 2627-638-1A), and the dashed line is for $T_{\text {eff }}=5800 \mathrm{~K}$ (i.e., for the derived temperature of the visual companion star TYC 2627-638-1B) at a common distance of 230 pc. The measured fluxes of TYC 2627-638-1B are far too low for the stellar temperature at the same distance, but a blackbody curve at a distance of about $400 \mathrm{pc}$, plotted with dotted line in Fig. 16, fits its $B V(R I)_{\mathrm{C}}$ fluxes well. The faintness of the star suggests some dust, which is further supported by the colour-magnitude diagrams in Fig. 12. Because we argue that the two stars as a bound system are at the same distance from us, and the brightness difference between the two components is more than one magnitude higher than follows from their respective spectral types and temperatures, the dust we look through towards the fainter star should be quite dense, like e.g. a nearly edge-on disk. More detailed imaging, also at the mid-infrared wavelengths, would help to study the environment of this system, including the third star TYC 2627-638-1C.
Concerning all measured and deduced evidence we think that TYC 2627-638-1A and TYC 2627-638-1B form a young, earlyG-type wide binary with two active components. A substellar companion TYC 2627-638-1Aa orbits the G0 star. Both stars, the more massive G0 component TYC 2627-638-1A as well as the fainter visual companion TYC 2627-638-1B with probably some remnant dust around, are close to the Young Main Sequence stars. This is strengthened by the fact that in Fig. 3. of Rojas et al. (2008), which is a plot of effective temperature vs. Li EWs of a sample of T Tau stars, post-T Tau stars and YMS stars from the Tucanae-Horologium association, TYC 2627-638$1 \mathrm{~A}$ and TYC 2627-638-1B are located among the YMS stars. Certainly, this interesting system needs further observational efforts to better describe the origin and properties of its components.

Acknowledgements. The authors are indebted to the two anonymous referees of this paper, whose suggestions and questions inspired us to get new important results and thus to improve the paper considerably. Our thanks are due to L. Balázs, L. L. Kiss, Z. Kiss, G. Kovács and B. Szeidl for helpful discussions. Nordic Optical Telescope is operated on the island of La Palma jointly by Denmark, Finland, Iceland, Norway, and Sweden, in the Spanish Observatorio del Roque de los Muchachos of the Instituto de Astrofisica de Canarias. ALFOSC is owned by the Instituto de Astrofisica de Andalucia and operated at the Nordic Optical Telescope under agreement between IAA and the Niels Bohr Institute for Astronomy, Physics and Geophysics of the Astronomical Observatory of Copenhagen. J.J., K.O. and K.V. acknowledges support from the Hungarian Research Grant OTKA T-068626.

\section{References}

Berdyugina, S. V. 1991, Bull. Crimean Astrophys. Obs., 83, 89 Bohlin, R. C., \& Gilliland, R. L. 2004, AJ, 128, 3053

Castelli, F., \& Kurucz, R. L. 2003, Proc. IAU Symp., 210, 20 Clarke, D. 2002, A\&A, 386, 763

Dall, T. H., Bruntt, H., \& Strassmeier, K. G. 2005, A\&A, 444, 573

Dall, T. H., Santos, N. C., Arentoft, T., Bedding, T. R., \& Kjeldsen, H. 2006, A\&A, 454, 341

Dias, W. S., Alessi, B. S., Moitinho, A., et al. 2002, A\&A, 389, 871

Donati, J.-F., Semel, M., Carter, B. D., Rees, D. E., et al. 1997, MNRAS, 291, 658

Famaey, B., Jorissen, A., Luri, X., et al. 2005, A\&A, 430, 165

Høg, E., Fabricius, C., Makarov, V. V., et al. 2000, A\&A, 355, L27

Ilyin, I. V. 2000, High-resolution SOFIN CCD échelle spectroscopy, Academic dissertation, University of Oulu

Jurcsik, J., Sódor, Á., Hurta, Zs., et al. 2008, MNRAS, 391, 164

Kupka, F., Piskunov, N., Ryabchikova, T. A., Stempels, H. C., \& Weiss, W. W. 1999, A\&AS, 138, 119

Kurucz, R. L. 1993, Kurucz CD No. 13

Mamajek, E. E., \& Hillenbrand, L. A. 2008, ApJ, 687, 1264

Meibom, S., Mathieu, R. D., \& Stassun, K. G. 2006, ApJ, 653, 621

Mentuch, E., Brandeker, A., van Kerkwijk, M. H., et al. 2008, ApJ, 689, 1127

Moór, A., Ábrahám, P., Derekeas, A., Kiss, Cs., et al. 2006, ApJ, 644, 525

Piskunov, N. E., Kupka, F., Ryabchikova, T. A., Weiss, W. W., \& Jeffrey, C. S. 1995, A\&AS, 112, 525

Royas, G., Gregorio-Hetem, J., \& Hetem, A. 2008, MNRAS, 387, 1335

Sbordone, L., Bonifacio, P., Castelli, F., et al. 2004, MemSS, 5, 93

Semel, M. 1989, A\&A, 225, 456

Šlechta, M., \& Škoda, P. 2002, Publ. Astron. Inst. ASCR, 90, 1

Schlegel, D. J., Finkbeiner, D. P., \& Davis, M. 1998, ApJ, 500, 525

Schröder, C., Reiners, A., Schmitt, J. H. M. M. 2009, A\&A, 493, 1099

Skrutskie, M. F., Cutri, R. M., Stiening, R., et al. 2006, AJ, 131, 1163

Siess, L., Dufour, E., \& Forestini, M. 2000, A\&A, 358, 593

Stetson, P. B., \& Pancino, E. 2008, PASP, 120, 1332

Zacharias, N., Finch, C., Girard, T., et al. 2010, ApJ, 139, 2184 